\title{
One-year Mortality of Cancer Patients with an Unplanned ICU Admission: A Cohort Analysis Between 2008 and 2017 in the Netherlands
}

Journal of Intensive Care Medicine I-9

(C) The Author(s) 2021 (c) (i)

Article reuse guidelines: sagepub.com/journals-permissions DOI: 10.1 I77/088506662II054369 journals.sagepub.com/home/jic (S)AGE

\author{
Esther N. van der Zee, MD' (D), Fabian Termorshuizen, PhD $^{2,3}$, \\ Dominique D. Benoit, MD, $\mathbf{P h D}^{4}$, Nicolette F. de Keizer, $\mathbf{P h D}^{2,3}$, \\ Jan Bakker, MD, PhD ${ }^{1,5,6,7}$, Erwin J.O. Kompanje, PhD', Wim \\ J.R. Rietdijk, PhD ${ }^{1, *}$, and Jelle L. Epker, MD, PhD ${ }^{1, *}$
}

\begin{abstract}
Introduction: A decrease in short-term mortality of critically ill cancer patients with an unplanned intensive care unit (ICU) admission has been described. Few studies describe a change over time of I-year mortality. Therefore, we examined the I-year mortality of cancer patients (hematological or solid) with an unplanned ICU admission and we described whether the mortality changed over time. Methods: We used the National Intensive Care Evaluation (NICE) registry and extracted all patients with an unplanned ICU admission in the Netherlands between 2008 and 2017. The primary outcome was I-year mortality, analyzed with a mixed-effects Cox proportional hazard regression. We compared the I-year mortality of cancer patients to that of patients without cancer. Furthermore, we examined changes in mortality over the study period. Results: We included 470,305 patients: I0,40 I with hematological cancer, 35,920 with solid cancer, and 423,984 without cancer. The I-year mortality rates were $60.1 \%, 46.2 \%$, and $28.3 \%$ respectively $(P<.01)$. Approximately $30 \%$ of the cancer patients surviving their hospital admission died within I year, this was $12 \%$ in patients without cancer. In hematological patients, I-year mortality decreased between 2008 and $20 \mathrm{I}$ I, after which it stabilized. In solid cancer patients, inspection showed neither an increasing nor decreasing trend over the inclusion period. For patients without cancer, I-year mortality decreased between 2008 and 20 I3, after which it stabilized. A clear decrease in hospital mortality was seen within all three groups. Conclusion: The I-year mortality of cancer patients with an unplanned ICU admission (hematological and solid) was higher than that of patients without cancer. About one-third of the cancer patients surviving their hospital admission died within I year after ICU admission. We found a decrease in I-year mortality until 20II in hematology patients and no decrease in solid cancer patients. Our results suggest that for many cancer patients, an unplanned ICU admission is still a way to recover from critical illness, and it does not necessarily lead to success in long-term survival. The underlying type of malignancy is an important factor for long-term outcomes in patients recovering from critical illness.
\end{abstract}

\section{Keywords}

intensive care unit, critical care, malignancy, cancer, neoplasm, ICU admission, mortality, survival, time trend, ICU triage

\section{Introduction}

Worldwide, approximately $40 \%$ of all people are diagnosed with cancer during their lifetime. ${ }^{1}$ In general, malignancies remain a major cause of death in Europe and North America. ${ }^{2,3}$ Fortunately, due to early detection and improved cancer treatment, long-term mortality of cancer patients has decreased over the past decades. ${ }^{1,4,5}$ However, the probability of life-threatening events related to therapy requiring intensive care has increased. ${ }^{6}$ Therefore, intensivists worldwide are increasingly confronted with cancer patients. ${ }^{7,8}$

Until the early 2000s, patients with advanced malignancy were generally perceived as ineligible for intensive care treatment due to the unfavorable outcomes. ${ }^{9-11}$ Nowadays, around $10 \%-20 \%$ of the patients admitted to an intensive care unit

\author{
'Erasmus University Medical Center, Rotterdam, the Netherlands \\ ${ }^{2}$ National Intensive Care Evaluation (NICE) foundation, Amsterdam, the \\ Netherlands \\ ${ }^{3}$ Amsterdam University Medical Center, Amsterdam Public Health research \\ institute, University of Amsterdam, Amsterdam, the Netherlands \\ ${ }^{4}$ Ghent University Hospital, Ghent, Belgium \\ ${ }^{5} \mathrm{New}$ York University, New York, USA \\ ${ }^{6}$ Columbia University Medical Center, New York, USA \\ ${ }^{7}$ Pontificia Universidad Católica de Chile, Santiago, Chile \\ *Shared last authorship.
}

Received July 6, 2021. Received revised September II, 2021. Accepted October 4, 2021.

Corresponding Author:

Esther van der Zee, MD, Department of Intensive Care, Erasmus MC University Medical Center, Room Ne-403, Doctor Molewaterplein 40, 3015 GD Rotterdam, the Netherlands.

Email: e.vanderzee@erasmusmc.nl 
(ICU) have cancer. ${ }^{12-14}$ Several studies show that ICU and hospital mortality of cancer patients has decreased over the years. ${ }^{9,14-19}$ In particular, a good prognosis in planned postsurgical cancer patients has been reported. ${ }^{8,15,18,20}$ Therefore, these patients are generally accepted to the ICU for postoperative care.

In contrast, studies reporting recent data regarding long-term mortality of cancer patients following unplanned ICU admission are scarce, especially studies describing a change over time of 1-year mortality. Recent data will complement previous literature, ${ }^{18-24}$ and is relevant for two reasons. First, in an acute setting, ICU physicians may be confronted with critically ill cancer patients. An enhanced understanding of outcomes in this patient group may lead to improved treatment decisions of hematologists, oncologists, and ICU physicians. Second, updated data may help to create realistic expectations toward patients and their relatives regarding ICU treatment and shortterm and long-term mortality.

Therefore, the aim of our study was to determine whether the observed decrease in short-term mortality of cancer patients with an unplanned ICU admission is associated with a decrease in 1-year mortality. We assessed 1-year mortality of patients with a malignancy (hematological or a solid cancer) and without malignancy with an unplanned admission to the ICU. Subsequently, we assessed whether there was a trend in 1 -year mortality over the study period.

\section{Methods}

\section{Patient Data}

The National Intensive Care Evaluation (NICE) registry was used to identify all adult patients with an unplanned admission to ICUs in the Netherlands from 2008 to 2017 . This registry contains data of patients from $85 \%$ of the Dutch ICUs in 2008 to $100 \%$ of the Dutch ICUs in $2017 .^{25}$ An unplanned ICU admission was defined as an admission due to an acute medical condition or an admission following scheduled surgery with intra-operative complications requiring ICU admission.

The NICE registry contains data on ICU admission (planned or unplanned), demographics, physiological and diagnostic data, ICU characteristics, and ICU and hospital mortality of patients. We linked a national administrative insurance claims database (Vektis) to the NICE registry, to determine the 1 -year mortality as previously reported. ${ }^{26,27}$ Health insurance is obligatory for all Dutch citizens and $99 \%$ have private healthcare insurance. ${ }^{28}$ The Vektis databases ${ }^{29}$ contain reimbursement data on all medical treatments paid for by Dutch insurance companies, as well as demographic information, such as date of birth, gender, and a proxy for date of death for all registered inhabitants of the Netherlands.

We stratified the patients into three cohorts: patients with hematological cancer, patients with a solid malignancy, and patients without cancer.
Patients with a hematological cancer were defined using the following three criteria: (i) patients referred from the hematological ward, (ii) patients with a hematological malignancy (e.g. leukemia or lymphoma) as admission diagnosis, or (iii) patients with "hematological malignancy" as comorbidity.

Patients with a solid malignancy were defined using the following three criteria: (i) patients referred from the oncology ward, (ii) patients with a solid malignancy as admission diagnosis, or (iii) patients with a comorbid condition "metastasized neoplasm".

The ICU patients without cancer were defined as all patients not included in the cohorts mentioned above.

All patients with a planned ICU admission were excluded. If patients were admitted to the ICU multiple times during the same hospitalization, only the first ICU admission was included in the analysis. Patients who underwent elective surgery and who subsequently had an unplanned ICU admission due to complications (e.g. after major bleeding during surgery) were included in this study as unplanned admissions.

\section{Baseline Characteristics and ICU Admission Characteristics}

A complete list of the characteristics and definitions can be found in Supplementary Table 1.

\section{Primary and Secondary Outcomes}

The primary outcome was 1-year mortality. Secondary outcomes were ICU and hospital mortality.

\section{Ethics}

The Medical Ethics Review Committee Erasmus Medical Center Rotterdam (decision number MEC-2019-0779) approved this study. Data are pseudonymized stored in the NICE registry and analyses were performed on an encrypted dataset. Because of the retrospective nature of the study and the usage of anonymous data, no additional informed consent was necessary. All procedures are in line with the General Data Protection Regulation (GDPR, May 2018) and law and regulations of the Netherlands.

\section{Statistical Analysis}

Baseline and ICU admission characteristics of the study population were reported as count (percentage) for categorical variables or as mean (standard deviation) for continuous variables. Differences between the three cohorts were analyzed with the chi-square test for categorical variables and the Wilcoxon test for continuous variables.

The 1-year mortality rates are reported as count and percentages for the three cohorts separately, differences were calculated with the chi-square test. We analyzed the 1-year mortality trend over the inclusion period using a mixed-effects 
Cox proportional hazard model with the calendar year 2008 to 2017 as an independent variable, using dummies with 2008 as reference. We used hospital as a random intercept. The models were adjusted for Acute Physiology and Chronic Health Evaluation (APACHE) IV mortality probability. Since the APACHE IV mortality probability also corrects for admission reasons besides age, physiology, and underlying illness, we chose APACHE IV mortality probability instead of the APACHE IV score. For this analysis, we presented hazard ratios (HRs) and 95\% confidence intervals (95\% CIs). Further, we used a post-estimation Wald test to examine whether there were statistically significant differences in 1 -year mortality over the inclusion period. This test examines the effect of including year dummies on mortality; however, it does not provide any information on whether there is a decreasing or increasing trend over the inclusion period. Therefore, we plotted the HRs and 95\% CIs in a graph. We performed a visual inspection on this graph, in which we compared HRs per calendar year to HRs in earlier years over the inclusion period, in order to determine whether a decreasing or increasing trend could be found. Moreover, when using a post-estimation Wald test, statistically significant differences and $P$-values refer to sample variation. Since we included all patients with an unplanned ICU admission to the Dutch ICUs during the study period, we did not use a sample. The post-estimation Wald test is therefore subordinate to the visual inspection.

The secondary outcomes were analyzed using a similar strategy, with the exception that a mixed-effects binary logistic regression analysis, with the calendar year (2008-2017) as an independent variable, was used. This model was adjusted for APACHE IV mortality probability and included a random intercept for the hospital. For this analysis, we present the odds ratios (OR) with their $95 \% \mathrm{CI}$. All analyses were performed using R-studio 3.6.1. A $P$ value of $<.05$ was considered statistically significant.

\section{Results}

\section{Baseline and Intensive Care Unit Admission Characteristics}

During the study period, 470,305 patients had an unplanned ICU admission, of these 10,401 (2.2\%) patients had hematological cancer, 35,920 (7.6\%) patients had a solid malignancy, and $423,984(90.2 \%)$ patients had no cancer. In Supplementary Table 2, an overview of missing values for all cohorts is reported.

Baseline and ICU admission characteristics of the three cohorts are shown in Table 1. All differences between the cohorts were statistically significant $(P<.05)$. The APACHE IV score was significantly higher in patients with hematological cancer compared to patients with a solid malignancy and patients without cancer, where there was no difference between the solid cancer and no-cancer patients (median 86 vs 59 and 57, respectively, $P<.01$ ). Within $24 \mathrm{~h}$ of ICU admission, $20.7 \%$ of the patients with hematological cancer had developed acute kidney failure (AKI), versus $10.2 \%$ in patients with a solid malignancy, and $11.1 \%$ in patients without cancer. Mechanical ventilation was used in $50.3 \%$ of the patients with hematological cancer, in $36.5 \%$ of the patients with solid cancer, and in $45.4 \%$ of patients without cancer. Vasoactive drugs were used in $47.2 \%$ of patients with hematological cancer, in $36.7 \%$ in patients with a solid malignancy, and in $34.6 \%$ of patients without a malignancy.

\section{Primary Outcome}

The 1-year mortality rate of patients with hematological cancer, a solid malignancy, and without cancer was $60.1 \%, 46.2 \%$, and $28.3 \%$, respectively $(P<.01$, Table 2$)$. Figure 1 A shows the trend of HRs for 1-year mortality over the study period for the three cohorts. For hematological patients, visual inspection of the 1-year mortality graph showed a decreasing trend until 2011, after which the mortality stabilized. The post-estimation Wald test showed no differences in mortality per year over the inclusion period $(P=.58)$. In contrast, for patients with a solid malignancy, the post-estimation Wald test showed a difference in 1-year mortality per year over the inclusion period $(P<.01)$. Visual inspection suggested this difference was mainly due to significantly lower HR in 2011, with a wide variation between HRs over other years, in neither an increasing nor decreasing trend. For patients without cancer, visual inspection of the 1-year survival graph suggested a decreasing trend in HRs until 2013, after which the mortality stabilized. The postestimation Wald test found a difference in 1-year mortality over the study period $(P<.01)$.

\section{Secondary Outcomes}

ICU mortality of hematological patients was $28.6 \%$, of patients with a solid malignancy $13.6 \%$, and of patients without cancer $12.5 \%(P<.01$, Table 2$)$. Figure 1B shows the trend of OR for ICU mortality over the study period for the three cohorts.

For patients with a hematological malignancy visual inspection suggested a decrease in ORs until 2012, with a slight increase from 2015 to 2017. The Wald test found a difference in ICU mortality over the inclusion period $(P<.01)$. For patients with a solid malignancy, visual inspection suggested a varying trend in HRs per year, with no clear decrease or increase. The Wald test found no difference in ICU mortality over the inclusion period $(P=.11)$. For patients without cancer visual inspection suggested a decreasing trend until 2013, after which the mortality stabilized. The Wald test found a difference in ICU mortality over the inclusion period $(P<.01)$.

The hospital mortality of hematological patients was $37.9 \%$, of patients with a solid malignancy $20.0 \%$, and of patients without cancer $16.4 \%,(P<.01)$. Figure $1 \mathrm{C}$ shows the trend of OR for hospital mortality over the inclusion period for the three cohorts. We found with a visual inspection a decrease in hospital mortality for each cohort over the inclusion period. The post-estimation Wald test suggested a difference for each of the three cohorts as well $(P<.05)$. 
Table I. Baseline and ICU Admission Characteristics.

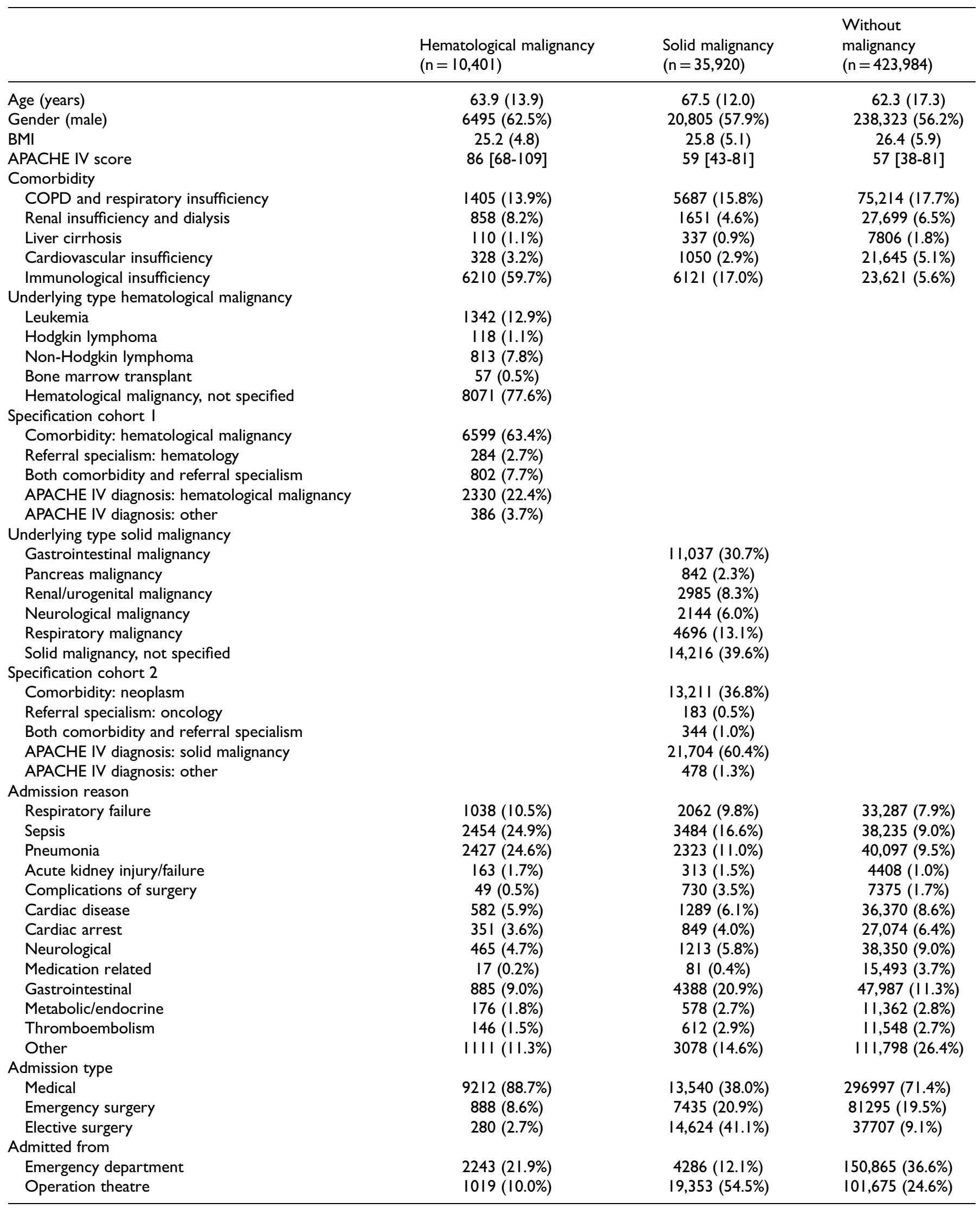


Table I. (continued)

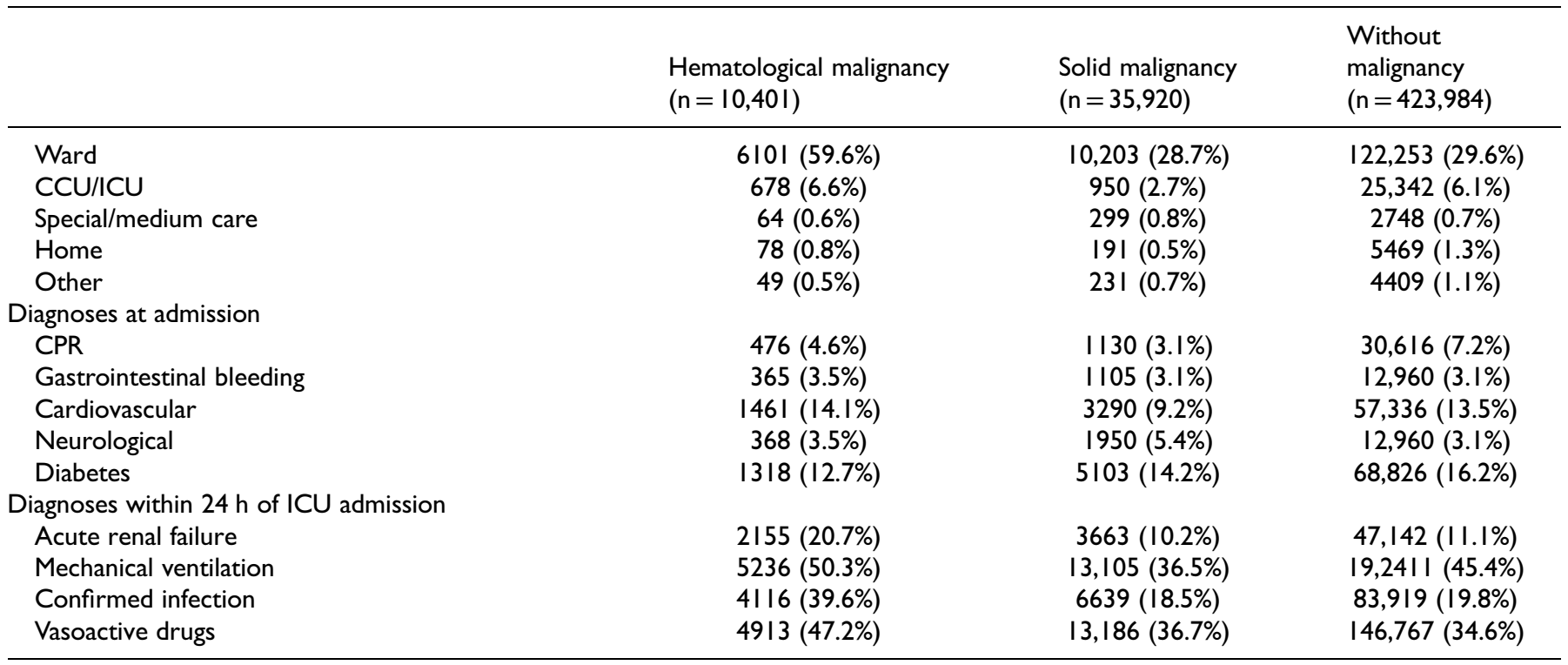

Data are displayed as mean (standard deviation) for continuous and count (percentages) for categorical variables, all $P$ values was highly significant with a $P<0.01$. Abbreviations: APACHE, Acute Physiology and Chronic Health Evaluation; BMI, body mass index; CCU, coronary care unit; CPR, cardiopulmonary resuscitation; COPD, chronic obstructive pulmonary disorder; ICU, intensive care unit.

\section{Additional Post Hoc Observations}

Three additional factors, relevant for the interpretation of our results, were also analyzed. First, when patients with cancer (either hematological or solid) survived hospital admission, approximately one-third died (30\%) within the year following that ICU admission. In patients without cancer, this number was lower (12\%).

Second, visual inspection of the APACHE IV probability of mortality over the study period suggested no major changes in illness severity at admission over the inclusion period for each cohort. The post-estimation Wald test showed differences in APACHE IV probability of mortality between calendar years over the inclusion period for all three cohorts $(p<.01)$. The APACHE IV probability of mortality for each cohort is presented in Supplementary Figure 1).

Third, the proportion of patients in the three cohorts was similar in each year during the study period. Patients with hematological malignancy represented $2 \%$ to $3 \%$, patients with a solid malignancy $7 \%$ to $8 \%$, and on average $90 \%$ of the admitted patients had no cancer (Supplementary Table 3).

\section{Discussion}

In this study, we showed that in the period of 2008 to 2017, cancer patients (either hematological or solid) with unplanned ICU admission had a significantly higher 1-year mortality compared to patients without cancer with an unplanned ICU admission. In patients with hematological cancer, we found a decrease in 1-year mortality from 2008 to 2011 , after which mortality stabilized. In patients with solid cancer, no change was seen. We found evidence for an overall decreasing trend in hospital mortality of critically ill cancer patients. Apparently, for many cancer patients, ICU admission is still a bridge to recover from their critical illness. It however does not necessarily lead to success in long-term survival, the underlying type of malignancy or its treatment is an important factor for long-term outcomes in patients recovering from critical illness.

In contrast to the decrease in long-term mortality over the past decades of cancer patients in general, ${ }^{1,4,5)}$ in this cohort of patients with an unplanned ICU admission, we found only a small decrease in 1-year mortality in hematological patients and no decrease in solid cancer patients. The variety in underlying types of malignancy and treatment options for those different malignancies might explain the slight or absent decrease in 1-year mortality of patients with cancer and an unplanned ICU admission. ${ }^{19}$ Interestingly, a recent study reporting mortality trends of septic shock patients showed a decrease in mortality between 2009 and 2011 after which it stabilized. ${ }^{30}$ In our study population, a considerable part (particularly in those with a hematological malignancy) was admitted with sepsis or infection to the ICU. The absence of new treatments since 2011 for sepsis and septic shock might explain the absence of a decrease in mortality after 2011.

Complementary to other literature, our study presents longterm mortality data from a nationwide registry in cancer patients with an unplanned ICU admission. ${ }^{18-24}$ Differences in case mix between our study and previous literature make direct comparison difficult. For example, a large study performed in the United States with data from 2002 to $2011^{19}$ reported a decrease in 1-year mortality of patients with hematological cancer and 
Table 2. Overview of Mortality Rates and ICU/Hospital Length of Stay.

\begin{tabular}{lccc}
\hline & $\begin{array}{l}\text { Hematological malignancy } \\
(\mathrm{n}=10,40 \mathrm{I})\end{array}$ & $\begin{array}{l}\text { Solid malignancy } \\
(\mathrm{n}=35,920)\end{array}$ & $\begin{array}{l}\text { Without malignancy } \\
(\mathrm{n}=423,984)\end{array}$ \\
\hline ICU mortality & $2969(28.6 \%)$ & $4890(13.6 \%)$ & $52,864(12.5 \%)$ \\
Hospital mortality & $3724(37.9 \%)$ & $6916(20.0 \%)$ & $65,026(16.4 \%)$ \\
I-year mortality & $5916(60.1 \%)$ & $15,606(46.2 \%)$ & $111,328(28.3 \%)$ \\
ICU length of stay (days) & $2.4[0.9-6.2]$ & $1.1[0.8-2.9]$ & $1.6[0.7-3.8]$ \\
Hospital length of stay after ICU (days) & $10.3[4.3-20.5]$ & $8.6[4.4-15.4]$ & $7.5[3.2-15.4]$ \\
\hline
\end{tabular}

All $P$-values assessing statistical differences between cohorts were highly significant with a $P<.01$.

Patients who were lost to follow-up are not included in this table.

Abbreviation: ICU, intensive care unit.

solid cancers. However, this study included both planned and unplanned ICU admissions. Interestingly, the final year of their database (2011) was similar to the year in which in our database, the decrease in 1-year mortality of hematological patients ended. Other studies regarding long-term mortality do not report trends over time. For example, Puxty et al. ${ }^{18}$ compared mortality rates of surgical patients with and without cancer admitted to the ICU. They reported a lower 1-year mortality when compared to our study, however, the majority of their patients had a planned ICU admission. Further, a study from Belgium ${ }^{20}$ showed comparable mortality for patients with hematological cancers to our study, while the 1-year mortality of patients with a solid malignancy was lower than in our study. Again, the study of Oeyen et al. included both planned and unplanned ICU patients. Two studies (Ehooman et al. ${ }^{24}$ and Azoulay et $\mathrm{al}^{31}$ ) included only hematological patients. ICU, hospital, and 1-year mortality rates of their patients were compared to that of the hematological patients in our study. Finally, the 1-year mortality of cancer patients in our study was lower than that of studies with smaller sample sizes from Spain, Austria, and Switzerland. ${ }^{21-23}$ Apart from sample size, there is only a small difference in case-mix between our study and these three studies. Although no decrease in 1-year mortality was seen in our study, the decrease in mortality when compared to these studies might be a reason for cautious optimism regarding long-term mortality.

As a secondary outcome, we found a decrease in hospital mortality over the inclusion period for all three cohorts. These results are in line with several other studies that showed a decreasing trend in hospital mortality of cancer patients. ${ }^{9,14-19}$ Relevant to note is that when cancer patients (either hematological or solid) survived the hospital admission, approximately one-third died within the year following the ICU admission. In patients without cancer, this number was considerably lower (12\%).

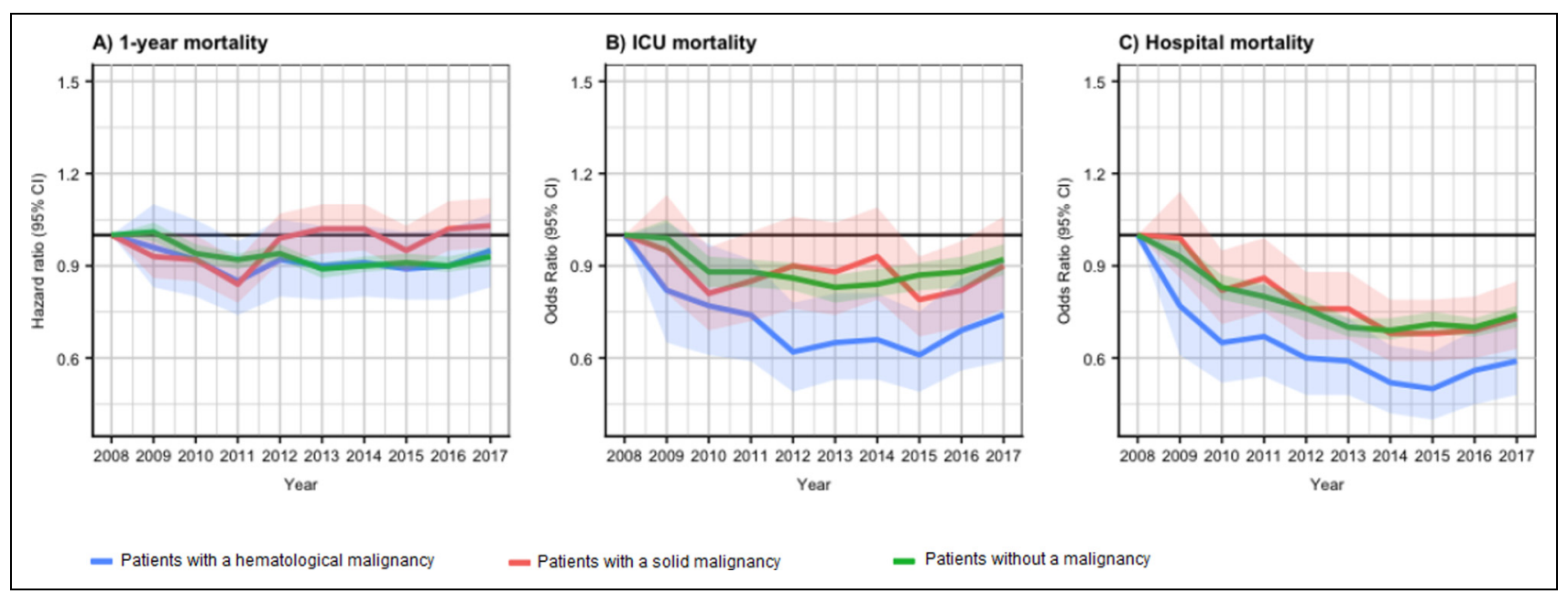

Figure I. (A) Shows the trend of hazard ratios (HRs) for I-year mortality over the inclusion period for the three cohorts (hematological patients, $P=.58$; solid malignancy, $P<.0 \mathrm{I}$; patients without a malignancy, $P<.0 \mathrm{I}$ ). (B) Shows the trend of odds ratios (OR) for ICU mortality over the inclusion period for the three cohorts (hematological patients, $P<.0 \mathrm{I}$; solid malignancy, $P=. \mathrm{II}$; patients without a malignancy, $P<.0 \mathrm{I}$ ). (C) Shows the trend of OR for hospital mortality over the inclusion period for the three cohorts (hematological patients, $P<.01$; solid malignancy, $P<.01$; patients without a malignancy, $P<.0$ I). $P$ values are based on a post-estimation Wald test. The actual hazard and odds ratios are available on request from the corresponding author.

Abbreviation: ICU, intensive care unit. 
One of the strengths of our study is that it adds to current literature. Especially, the fact that the underlying type of malignancy or its treatment may be an important factor for long-term outcomes in patients recovering from critical illness. A large recent study ${ }^{32}$ showed similar results. Among all diagnostic subgroups of the ICU population included in that study, patients with nonsurgical cancer had the lowest cumulative 1-year survival. A possible explanation could be that a considerable part of the deaths within one year in the cancer cohorts is directly or indirectly related to the underlying malignancy or its treatment. This may explain the clear improvement in short-term mortality of cancer patients, while no clear improvement in long-term mortality was seen. A possible and not unlikely explanation for the decrease in hospital mortality and high 1-year mortality may be the physician's decision to discharge patients to a nursing home or hospice for the terminal phase, leading to a spurious reduction of mortality risk when using hospital death as an outcome. ${ }^{33,34}$ Unfortunately, we have no data available in the NICE register to support this hypothesis. However, ICU admissions of patients are often determined by short-term survival benefit, and not by long-term prognosis. ${ }^{35}$ In addition, physicians do not always communicate adequately about the prognosis and benefits of treatments toward patients and relatives. ${ }^{36-38}$ Ideally, oncologists and hematologists discuss long-term outcomes after an ICU admission with patients and relatives well before an ICU admission, in order to manage expectations. All physicians should consider the benefits and burden of an ICU admission for each patient individually before ICU referral or admission.

\section{Limitations}

For interpreting the study results, the following limitations should be considered.

First, our study is a general description of a heterogeneous population of critically ill cancer patients (both hematological malignancies and solid malignancies) with an unplanned ICU admission.

Second, we could not include the type and stage of the malignancy, the continuation of cancer treatment, and the performance status prior to ICU admission. Originally, the NICE registry is a quality of ICU care registry in the Netherlands, in which not all clinically relevant factors for cancer patients are registered. However, the impact on the outcomes of the current study might be limited as it is unlikely that cancer types and stages or performance status have significantly changed during the study period. ${ }^{39}$

Third, various reasons ICU admission exists in cancer patients. These factors were beyond the scope of this descriptive study and although relevant, cannot be reported.

Fourth, we performed a post-estimation Wald test and a visual inspection. To interpret our results, we used the visual inspection as the leading result. As described in the methods, the post-estimation Wald test is subordinate to the visual inspection.
Lastly, differences in treatment standards may occur for cancer patients across hospitals. However, by including a hospital random intercept in our models, we corrected for hospital variations. In addition, the actual numbers and proportions of cancer patients with an unplanned ICU admission were not different over the study period. This suggests that at least similar numbers of cancer patients were admitted without structural changes in admission policies.

\section{Future Research}

Linking the NICE registry with the national cancer registry in the Netherlands may enrich the data with clinically relevant factors. Such enrichment may help to identify patients with a malignancy who are likely to benefit most from ICU admission. ${ }^{6,7}$

It would be helpful to establish more uniform inclusion criteria to enable a viable comparison between countries. To minimize other sources of heterogeneity, analysis on subgroups of cancer patients, such as septic and nonseptic patients, may be considered in future research.

\section{Conclusion}

The 1-year mortality of cancer patients (hematological and solid) with an unplanned ICU admission is higher than that of patients without cancer. One-third of the cancer patients surviving ICU admission died within 1 year after ICU admission. We found a decrease in 1-year mortality until 2011 in hematology patients and no decrease in solid cancer patients. Our results suggest that in many cancer patients, an unplanned ICU admission is still a bridge to recover from critical illness, and it does not necessarily lead to success in long-term survival. The underlying type of malignancy is an important factor for long-term outcomes in patients recovering from critical illness.

\section{Abbreviations}

AKI acute kidney failure

APACHE Acute Physiology and Chronic Health Evaluation

BMI body mass index

$\mathrm{CCU} \quad$ coronary care unit

95\% CIs $\quad 95 \%$ confidence intervals

COPD chronic obstructive pulmonary disease

CPR cardiopulmonary resuscitation

HR hazard ratio

ICU intensive care unit

LOS length of stay

NICE National Intensive Care Evaluation

OR odds ratio.

\section{Acknowledgments}

None. 


\section{Author Contributions}

EZ: conceptualization, design, interpretation of data, writing —original draft and writing - review and editing, FT: design, analysis and interpretation of data, and writing - review and editing. DB: interpretation of data, writing - review and editing, NK: design, analysis and interpretation of data, and writing - review and editing, JB: interpretation of data, and writing - review and editing EK: interpretation of data, and writing-review and editing, WR: conceptualization, design, analysis and interpretation of data, writing-original draft and writing - review and editing, JE: conceptualization, design, analysis and interpretation of data, writing-original draft and writingreview and editing. WR and JE are Joint last authors. All authors read and approved the final manuscript.

\section{Availability of Data and Materials}

The data of this study are available from the National Intensive Care Evaluation register. Restrictions apply to the availability of these data, which were used under license for the current study, and are not publicly available. Data are available from the authors upon reasonable request and with permission of the National Intensive Care Evaluation register.

\section{Declaration of conflicting interests}

The author(s) declared no potential conflicts of interest with respect to the research, authorship, and/or publication of this article.

\section{Funding}

The author(s) received no financial support for the research, authorship, and/or publication of this article.

\section{Ethical Approval}

Not applicable, because this article does not contain any studies with human or animal subjects.

\section{ORCID iD}

Esther N. van der Zee (iD https://orcid.org/0000-0003-0431-4659

\section{Supplemental Material}

Supplemental material for this article is available online.

\section{References}

1. National Cancer Institute. 2016. updated 13 September 2018. https://seer.cancer.gov/statfacts/html/all.html.

2. Eurostat Statistics. 2015. updated 7 September 2018. http://ec. europa.eu/eurostat/statistics-explained/index.php/Causes_of_deat h_statistics.

3. Wang H, Naghavi M, Allen C, et al. Global, regional, and national life expectancy, all-cause mortality, and cause-specific mortality for 249 causes of death, 1980-2015: a systematic analysis for the global burden of disease study 2015. Lancet. 2016;388(10053):1459-1544

4. Allemani C, Matsuda T, Di Carlo V, et al. Global surveillance of trends in cancer survival 2000-14 (CONCORD-3): analysis of individual records for 37513025 patients diagnosed with one of 18 cancers from 322 population-based registries in 71 countries. Lancet. 2018;391(10125):1023-1075.
5. Malvezzi M, Carioli G, Bertuccio P, et al. European cancer mortality predictions for the year 2016 with focus on leukaemias. Ann Oncol. 2016;27(4):725-731.

6. Azoulay E, Pene F, Darmon M, et al. Managing critically Ill hematology patients: time to think differently. Blood Rev. 2015;29(6):359-367.

7. Azoulay E, Schellongowski P, Darmon M, et al. The intensive care medicine research agenda on critically ill oncology and hematology patients. Intensive Care Med. 2017;43(9):1366-1382.

8. Shimabukuro-Vornhagen A, Boll B, Kochanek M, Azoulay E, von Bergwelt-Baildon MS. Critical care of patients with cancer. CA Cancer J Clin. 2016;66(6):496-517.

9. Ostermann M, Ferrando-Vivas P, Gore C, Power S, Harrison D. Characteristics and outcome of cancer patients admitted to the ICU in england, wales, and northern Ireland and national trends between 1997 and 2013. Crit Care Med. 2017;45(10):1668-1676.

10. Guidelines for intensive care unit admission, discharge, and triage. Task force of the American college of critical care medicine, society of critical care Medicine. Crit Care Med. 1999;27(3):633-638.

11. Garrouste-Orgeas M, Montuclard L, Timsit JF, et al. Predictors of intensive care unit refusal in French intensive care units: a multiple-center study. Crit Care Med. 2005;33(4):750-755.

12. Soares M, Caruso P, Silva E, et al. Characteristics and outcomes of patients with cancer requiring admission to intensive care units: a prospective multicenter study. Crit Care Med. 2010;38(1):9-15.

13. Taccone FS, Artigas AA, Sprung CL, Moreno R, Sakr Y, Vincent JL. Characteristics and outcomes of cancer patients in european ICUs. Crit Care. 2009;13(1):R15.

14. Zampieri FG, Romano TG, Salluh JIF, et al. Trends in clinical profiles, organ support use and outcomes of patients with cancer requiring unplanned ICU admission: a multicenter cohort study. Intensive Care Med. 2020;47(2):170-179.

15. Bos MM, de Keizer NF, Meynaar IA, Bakhshi-Raiez F, de Jonge E. Outcomes of cancer patients after unplanned admission to general intensive care units. Acta Oncol. 2012;51(7):897-905.

16. Darmon M, Bourmaud A, Georges Q, et al. Changes in critically ill cancer patients' short-term outcome over the last decades: results of systematic review with meta-analysis on individual data. Intensive Care Med. 2019;45(7):977-987.

17. Huaringa AJ, Francis WH. Outcome of invasive mechanical ventilation in cancer patients: intubate or not to intubate a patient with cancer. J Crit Care. 2019;50:87-91.

18. Puxty K, McLoone P, Quasim T, Sloan B, Kinsella J, Morrison DS. Characteristics and outcomes of surgical patients With solid cancers admitted to the intensive care unit. JAMA Surg. 2018;153(9):834-840.

19. Sauer CM, Dong J, Celi LA, Ramazzotti D. Improved survival of cancer patients admitted to the intensive care unit between 2002 and 2011 at a U.S. Teaching hospital. Cancer Res Treat. 2019;51(3):973-981.

20. Oeyen SG, Benoit DD, Annemans L, et al. Long-term outcomes and quality of life in critically ill patients with hematological or solid malignancies: a single center study. Intensive Care Med. 2013;39(5):889-898.

21. Bernal T, Pardavila EV, Bonastre J, et al. Survival of hematological patients after discharge from the intensive care unit: a prospective observational study. Crit Care. 2013;17(6):R302.

22. Staudinger T, Stoiser B, Mullner M, et al. Outcome and prognostic factors in critically ill cancer patients admitted to the intensive care unit. Crit Care Med. 2000;28(5):1322-1328. 
23. Unseld S, Schuepbach RA, Maggiorini M. ICU, hospital and one year mortality of patients suffering from solid or haematological malignancies. Swiss Med Wkly. 2013;143:w13741.

24. Ehooman F, Biard L, Lemiale V, et al. Long-term health-related quality of life of critically ill patients with haematological malignancies: a prospective observational multicenter study. Ann Intensive Care. 2019;9(1):2.

25. Mandigers L, Termorshuizen F, de Keizer NF, et al. A nationwide overview of 1-year mortality in cardiac arrest patients admitted to intensive care units in the Netherlands between 2010 and 2016. Resuscitation. 2020;147:88-94.

26. van de Klundert N, Holman R, Dongelmans DA, de Keizer NF. Data resource profile: the Dutch national intensive care evaluation (NICE) registry of admissions to adult intensive care units. Int $J$ Epidemiol. 2015;44(6):1850-h.

27. Mandigers L, Termorshuizen F, de Keizer NF, et al. Higher 1-year mortality in women admitted to intensive care units after cardiac arrest: a nationwide overview from the Netherlands between 2010 and 2018. J Crit Care. 2021;64:176-183.

28. Statistics Netherlands' database: Statistics Netherlands (CBS). updated 2021. https://opendata.cbs.nl/statline/\#/CBS/en/.

29. Vektis. Vektis: Vektis. 2021. updated 20021. https://www.vektis.nl/.

30. Bauer M, Gerlach H, Vogelmann T, Preissing F, Stiefel J, Adam D. Mortality in sepsis and septic shock in Europe, North America and Australia between 2009 and 2019- results from a systematic review and meta-analysis. Crit Care. 2020;24(1):239.

31. Azoulay E, Mokart D, Pene F, et al. Outcomes of critically ill patients with hematologic malignancies: prospective multicenter data from France and Belgium--a groupe de recherche respiratoire en reanimation onco-hematologique study. J Clin Oncol. 2013;31(22):2810-2818
32. van Valburg MK, Termorshuizen F, Brinkman S, et al. Long-Term mortality Among ICU patients With stroke compared With other critically Ill patients. Crit Care Med. 2020;48(10): e876-ee83.

33. Pouw ME, Peelen LM, Moons KG, Kalkman CJ, Lingsma HF. Including post-discharge mortality in calculation of hospital standardised mortality ratios: retrospective analysis of hospital episode statistics. Bmj. 2013;347(7931):f5913.

34. Cohen J, Pivodic L, Miccinesi G, et al. International study of the place of death of people with cancer: a population-level comparison of 14 countries across 4 continents using death certificate data. Br J Cancer. 2015;113(9):1397-1404.

35. Escher M, Nendaz M, Scherer F, Cullati S, Perneger $T$. Physicians' predictions of long-term survival and functional outcomes do not influence the decision to admit patients with advanced disease to intensive care: a prospective study. Palliative Medicine. 2020;35(1):161-168.

36. Audrey S, Abel J, Blazeby JM, Falk S, Campbell R. What oncologists tell patients about survival benefits of palliative chemotherapy and implications for informed consent: qualitative study. $\mathrm{Bmj}$. 2008;337(7668):a752.

37. Lamont EB, Christakis NA. Prognostic disclosure to patients with cancer near the end of life. Ann Intern Med. 2001;134(12):10961105 .

38. Weeks JC, Catalano PJ, Cronin A, et al. Patients' expectations about effects of chemotherapy for advanced cancer. $N$ Engl J Med. 2012;367(17):1616-1625.

39. Borcoman E, Dupont A, Mariotte E, et al. One-year survival in patients with solid tumours discharged alive from the intensive care unit after unplanned admission: a retrospective study. J Crit Care. 2020;57:36-41. 